\title{
Significados del cuerpo en personas con Diabetes Mellitus en la ciudad de Cali*
}

\author{
Maritza Charry Higueras ${ }^{* *}$
}

Recibido: diciembre 5 de 2015 • Evaluado: enero 20 de 2016

Aceptado: febrero 8 de 2016

\section{Resumen}

La presente investigación surgió como parte de las experiencias de intervención familiar en salud. Se realizó con cuatro personas diagnosticadas con diabetes mellitus con el fin de conocer los significados del cuerpo a partir de la enfermedad y de las relaciones con personas relevantes, incluyendo el médico. Su metodología fue cualitativa y se desarrolló a través de entrevistas a profundidad. Entre los principales resultados se destaca el cuerpo significado como algo constitutivo, temporal y físico que permite el acceso al mundo; cómo un dispositivo de acción simbólica, de comunicación y de lenguaje humano se construye en la relación con el otro, con quien se consolida nuestra identidad e imagen corporal regulada estéticamente. Se concluye que cuando el cuerpo enferma, la experimentación corporal y la resultante noción del cuerpo cambian no slo por las sensaciones corporales y subjetivas del ser humano, sino por las construcciones sociales que se hacen del cuerpo y de la enfermedad.

Palabras clave: cuerpo; enfermedad; significado; diabetes mellitus

* El siguiente artículo es producto de la ponencia presentada en el II Encuentro latinoamericano de investigadores sobre el cuerpo, realizado entre el 3 y el 7 de octubre de 2015 en la ciudad de Bogotá - Colombia. La investigación se titulo: "El cuerpo, entre la salud y la enfermedad: Significados del cuerpo en personas con Diabetes Mellitus en la ciudad de Cali" y se realizó para optar al titulo de magíster en Intervención Social - Campo de Profundización en Familia por la Universidad del Valle. La investigación completa y una ampliación del tema de la intervención constructivista en salud serán publicadas más adelante en un capítulo de libro. DOI: http://dx.doi.org/10.15332/s2248-4418.2016.0001.02

** Magíster en Intervención Social - Campo de Profundización en Familia; especialista en Intervención con Familias; docente de la Universidad del Valle en la Facultad de Humanidades. Escuela de Trabajo Social y Desarrollo Humano. Correo electrónico: maritza.charry@ correounivalle.edu.co 


\title{
Meanings of the body in peoble with diabetes mellitus in the city of Cali
}

\begin{abstract}
This investigation arose as part of the experiences of family health interventions; it was performed with four people diagnosed with diabetes mellitus seeking to know the meanings of the body from disease and relationships with significant others, including the doctor. Methodology was qualitative and developed through in-depth interviews. The main results highlighted the body meaning as constitutive, temporal and physical that allows access to the world and as a device symbolic action, communication and human language, is built on the relationship with the other, with whom our identity is consolidated and aesthetically regulated body image. It concluded that when the body is sick, body experimentation and the resulting notion of it changes, not only for the physical and subjective feelings of human beings, but by the social constructions that are made of body and disease.
\end{abstract}

Keywords: body; disease; meaning 


\section{Introducción}

Las principales tendencias teóricas que se han venido utilizando en el estudio del cuerpo como objeto de conocimiento se pueden resumir en cuatro. La primera obedece a los desarrollos filosóficos de Descartes, quien fundó la visión dicotómica del cuerpo y dio origen a las diversas teorías sobre el ser humano que dividen el estudio mental y emocional de la corporalidad (Ramfis, 2001). Sin embargo, esta tendencia es confrontada con los trabajos fenomenológicos del filósofo francés Merleau-Ponty (1984), quien reivindica el cuerpo en su carácter constitutivo de existencia y experimentación del mundo, es decir, es a través de los sentidos corporales que percibimos y experimentamos la existencia del mundo. En esta misma línea se encuentran los trabajos contemporáneos de Kleinman (1988), Das (1996), Le Bretón (2007) y Citro (2009), quienes retoman la visión fenomenológica de la corporalidad para aplicarla en contextos de salud, violencia y sufrimiento humano.

La segunda tendencia se refiere a los aportes de Foucault (1966; 2006) y Turner (1988) al considerar que el cuerpo recibe el peso de las estructuras sociales y que la mirada clínica ejerce un poder sobre el cuerpo y los procesos de salud - enfermedad a través del discurso médico, obligando al profesional a reducir el cuerpo a un objeto de observación. En esta tendencia se ubican los desarrollos recientes de Suárez (2001), Pedraza (2003) y Sierra (2009).

La tercera tendencia correspondería a la línea civilizatoria del cuerpo: aquellos aportes de Mauss (1979) y Elías (1966) en los que consideran que el cuerpo se convierte en un instrumento técnico donde se determinan formas de actuar y comportarse, las llamadas técnicas corporales, modales transmitidos por la educación y "ordenados" por la sociedad para comportarse dentro de las lógicas que las sociedades construyen.

Finalmente, en una última tendencia en el estudio del cuerpo, se ubican las investigaciones de De Certeau (2000). El autor afirma que se pueden reconfigurar las determinaciones sociales que recibe el cuerpo a partir de la creación de prácticas cotidianas; considera que todos los seres humanos tenemos ciertos grados de autonomía y libertad para posibilitar una segunda fabricación como una forma de resistir a tales determinaciones sociales.

Teniendo en cuenta las anteriores tendencias, la investigación se fundamentó en la articulación de la perspectiva fenomenológica del cuerpo, 
retomando los postulados de Ponty (1984) acerca de la percepción corporal del mundo y la perspectiva constructivista (Turner, 1989) que reconoce al cuerpo como una construcción social en permanente interacción con las determinaciones que promueven las estructuras sociales, el discurso médico y las relaciones de convivencia.

La presente investigación partió de experiencias de intervención terapéutica con personas con diabetes y pretendió profundizar en los procesos salud - enfermedad como objeto de conocimiento; específicamente se buscó comprender los significados del cuerpo construidos por dos hombres y dos mujeres ante el diagnóstico de la enfermedad crónica de la diabetes mellitus tipo II, partiendo de las relaciones de convivencia con personas significativas. Se partió de la hipótesis de considerar que entre mayor fuera el tiempo de diagnóstico de la diabetes, las modificaciones en los estilos de vida de los sujetos serían más notables, además de que las relaciones de convivencia con las personas significativas influían de alguna manera en la construcción de los significados que hacían sobre la enfermedad y su cuerpo.

\section{Metodología}

Coherente con la metodología cualitativa de la investigación, se utilizó como estrategia el estudio de caso y como técnica la entrevista a profundidad. Si bien la ejecución de esta investigación no se enmarcó dentro de un contexto institucional, las cuatro personas entrevistadas (dos hombres y dos mujeres, entre los 37 años y los 69 años) tenían relación con algún tipo de control médico dentro de las instituciones de salud que los atendían. Su selección dependió de el interés de participar, la ubicación en rangos de edad distintos y un tiempo superior a tres meses de diagnosticada la diabetes mellitus tipo II. En total, se realizaron diez entrevistas a los cuatro participantes; de ellas, seis fueron individuales y cuatro se realizaron con las personas con quienes ellos convivían. Las entrevistas transcritas fueron entregadas posteriormente a cada uno de ellos para su lectura y retroalimentación de la experiencia. Posterior a la realizacion de las entrevistas, se realizó el análisis de las categorias de análisis construidas inicialmente: significado del cuerpo ante la enfermedad crónica, influencia de las relaciones de convivencia y de la mirada médica en la construcción de los significados alrededor del cuerpo. 


\section{Resultados}

La investigación se fundamentó en las perspectivas teóricas fenomenológicas de Ponty (1984), que hacen un acercamiento constitutivo al cuerpo en la materialización de las enfermedades; de las perspectivas constructivistas planteadas por Turner (1989) y Foucault $(1966 ; 1990)$, que reconocen el cuerpo como una construcción social en permanente relación tanto con las determinaciones que promueven las estructuras sociales, como con las posibilidades de lucha y creación autónoma que tienen las personas, estudiados por De Certeau (2000). Tales perspectivas ayudaron a comprender el cuerpo desde la convergencia de polos que hasta ahora han venido tratándose de manera aislada. Además, se consideró la enfermedad desde una mirada sociológica como un proceso que combina tres nociones cardinales: "1) la enfermedad es un lenguaje, 2) el cuerpo es una representación, y 3) la medicina es una práctica política” (Turner, 1989, p. n253). Considerar la enfermedad como un lenguaje es verla como un proceso que se construye socialmente a partir de una base biológica connotada subjetivamente. La enfermedad es social porque representa, en un lenguaje metafórico corporal, algo propio, único y particular de la historia de cada persona, movilizándola hacia la reflexión de las relaciones sociales y la ejecución de acciones.

A partir de las entrevistas construidas con las cuatro personas, se encontró diferencias en edad, nivel educativo, estrato socioeconómico, estado civil, momento de su vida y tiempo de diagnóstico de la enfermedad (entre uno y doce años). Se hallaron dos nociones corporales: una asociada a la enfermedad y la otra movilizada por el discurso médico; ambas complementarias.

\section{La noción del cuerpo ante la enfermedad}

En las cuatro personas, la noción del cuerpo fue diversa, tanto en su concepción como en la forma de experimentarlo, evidenciándose, como lo plantea Soler (2003), que no hay sujeto sin cuerpo, pues somos seres corporalizados cuya imagen se modifica continuamente en la vida y se reconstruye no en solitario, sino en relación con el juicio y la mirada que hace el otro sobre la imagen corporal propia; el Otro es la fuente principal de aprobación, de cuidado y del deber ser corporal. 
Las personas definieron el cuerpo como algo "fundamental, preciado y principal que hay que cuidar y querer"; como algo constitutivo e imprescindible para nuestra existencia, al que se le atribuye una definición valorativa. También consideraron el cuerpo como un vehículo de comunicación humana que se usa "para comunicarse con las personas". Además de la dimensión física, el cuerpo expresa una dimensión social de la historia de sus relaciones y de las experiencias de dolor y sufrimiento vivido, de las cuales no ha podido hacerse cargo emocionalmente. Esto lo confirma Kleinman (1988) cuando menciona que el lenguaje corporal es el lenguaje de las relaciones. En el caso de la primera persona entrevistada -Emma- se encontró que surgían sensaciones dolorosas no solo por el deterioro del cuerpo por el avance de la enfermedad de la diabetes, sino por la relación que estableció con los otros, construyéndose una percepción también dolorosa del mundo: "empezó a dormírseme un dedo, luego toda la pierna, casi hasta la rodilla [...] me duele la piel, me duelen los huesos, hasta el busto, no resisto [...] vivo sola con la empleada del servicio, eventualmente vienen por unos días mis hijos de Bogotá [...] siempre es como que me falta algo, me falta alguien, y eso siento que me hace daño". En su caso, el dolor y el sufrimiento que le representa vivir con su enfermedad reflejaron su condición histórico-social, como lo manifiesta Kleinman (1988, p. 13): "todo dolor comporta un padecimiento moral" y "un cuestionamiento de las relaciones entre el hombre y el mundo" Ante la enfermedad, el significado del cuerpo cambia tanto en la manera de concebirlo como de cuidarlo, promoviéndose la reflexión sobre los vínculos establecidos con las personas más significativas: madre, hermanos, esposos, hijos, cónyuges.

Un aspecto relevante encontrado hace referencia al temor por el deterioro del cuerpo ante la diabetes, tanto mujeres como hombres vivieron la enfermedad como algo

Silencioso, que va acabando con todo. (Emma)

Oculto y secreto que uno no sabe cuándo le va a disparar algo. (Esposa de Roberto)

Un mal que no siento, un enemigo oculto. (Simón)

Un cáncer [...] a medida que va pasando el tiempo, si yo no hago cosas eso va a avanzar más rápido. (Roberto) 
El tema del deterioro del cuerpo es definitivo para comprender los temores y miedos asociados con la enfermedad y significarla como algo horroroso. Como lo plantearon las personas entrevistadas, en ocasiones una enfermedad grave puede sentirse como una traición a la confianza básica en nuestros cuerpos (Kleinman, 1988), pues ataca de manera silenciosa. La enfermedad se vive con una existencia propia e independiente de nosotros mismos. El deterioro se puede comprender como una "alteración no deseada del metabolismo" (Turner, 1989, p. 281), cuyas consecuencias han sido ampliamente estudiadas por la medicina, debido a las complicaciones clínicas y al daño que causa la afección en el cuerpo.

\section{La noción del cuerpo ante el discurso médico}

El discurso médico logra separar al sujeto de su cuerpo, concibiéndolo como un organismo biológico, que olvida a la persona, sus sentimientos y relaciones sociales. Concibe el cuerpo como un "cuerpo humano dividido en trozos" que se estudia por un saber experto y se ocupa solamente de órganos, funciones y patologías:

El cuerpo lo defino como el conjunto de órganos que trabaja en equipo para permitir nuestro desarrollo sicosocial. La diabetes mellitus es un grupo de enfermedades que se caracterizan por la elevación persistente de la glucemia, con complicaciones crónicas sistémicas (en todo el cuerpo) si no hay control [...] afecta todo el organismo (el cuerpo) disminuyendo o alterando la función de cada uno (o de todos) de los órganos, con disminución de la calidad de vida de la persona, si no hay control. Les digo a los pacientes que es una enfermedad crónica que debe estar en buen control (sin daños a nuestro cuerpo) o con complicaciones agudas o crónicas. Les informo que ese curso depende no solo de los tratamientos instaurados y recomendaciones del equipo de salud, sino también del aporte en los estilos de vida saludable y la actitud a lo largo de la vida; si hay buena actitud, habrá más probabilidades de control. (Dr. Alberto)

Parafraseando a Foucault (1966), las maneras de pensar y la información que la medicina entrega a las personas, ejercen un poder de convencimiento, condicionamiento, regulación y control sobre sus pensamientos, sentimientos y acciones sobre sus cuerpos. 
Las personas sienten la obligación de asistir a las citas médicas para reconocer su cuerpo y lo que les pasa, legitimando en algún sentido el escrutinio del cuerpo y la invasión a su intimidad: "A mí me toca por obligación [...] estar llevando muestras de sangre o voy donde el médico a que me revise cómo estoy" (esposo de Patricia). Como lo plantearon Roberto y Patricia, este discurso médico determinó que fueran a consulta cuando presentaron sensaciones corporales, no regulares en su vida corriente, que son denominadas sintomas o formas "bajo la cual se presenta la enfermedad" y que permiten "designar un estado patológico (por oposición a la salud)..." (Foucault, 1966, p. 131):

Cuando comencé tenía los síntomas [...] tenía cansancio temporal, me dolían las articulaciones, me cansaba fácilmente y tenía resequedades en la boca; yo no sabía que era por el tema de la diabetes [...] entonces fui donde el médico. (Roberto)

Yo fui un día porque estaba manejando y me dio mucho el reflejo en los ojos y no vi $[\ldots]$ entonces a mí se me empezó a hacer raro y ahí fue cuando me descubrieron lo de la diabetes. (Patricia)

En este contexto, el cuidado del cuerpo no surge como un deseo personal, sino como un mandato del médico, con un carácter de deber que evita el placer y reprime el deseo. En las mujeres, el cuidado es significado como una forma de "querer su cuerpo", inicia con la higiene diaria, con las acciones de cuidado de la piel y el rostro. Para los cuatro participantes, cuidar el cuerpo tuvo que ver con una alimentación balanceada, realizar ejercicio físico, asistir a los "controles" médicos y tomarse los medicamentos. El cuidado del cuerpo es hacer lo que digan los médicos porque está en juego la salud y la vida.

Pero también, el discurso médico contiene la trampa de la maximización del poder del control del cuerpo, ubicando el control al interior del sujeto, dejándose de lado los demás componentes de la vida misma. Se encontró que el discurso por el cuidado del cuerpo afectó la relación médicopersona, estableciéndose dos tipos de relación:

- Relaciones de confianza: cuando las personas fueron tenidas en cuenta y el médico pudo ser abierto e integrarse a ellos y la familia. Se añora el rol del médico familiar: dedicado, con tiempo para conocer y escuchar a las personas. 
- Relación de desconocimiento: cuando las personas expresaron críticas frente a la atención recibida, el haberse sentido "como una ficha porque se dan recetas a todos por igual. Se burlan de lo que uno dice. Hacen apuestas con nosotros a ver quién mejora. El médico es arrogante/ pedante. El médico no saluda".

Se confirma lo que plantea Le Bretón (1999): la relación entre el profesional y la persona tiene el poder de influir en la evolución de la enfermedad, casi como sucede con el efecto placebo, moviliza el vínculo social con un otro que lo ayuda a poner remedio o alivio al sufrimiento. El efecto placebo aduce a todas aquellas situaciones y circunstancias posteriores al diagnóstico de una enfermedad que modifican su curso en términos de "síntomas, signos u otros indicadores que experimenta el organismo, que no es atribuible al mecanismo de acción específico de una terapéutica, ya sea mecánica, farmacológica, quirúrgica, ambiental o debida a cualquier otra situación curativa" (Tempone, 2007, p. 250); aunque también pueden ocurrir reacciones adversas que empeoran el curso de la enfermedad, lo que se denomina efecto nocebo.

La relación profesional permite aliviar o no su sufrimiento a través del afecto o rechazo que recibieron las personas en la consulta médica, dependiendo del control de la enfermedad. Por ejemplo: cuando hubo control de la enfermedad, los sujetos recibieron felicitaciones y el médico se mostró receptivo. Los sujetos se ubicaron en un plano personal para iniciar los procesos de cambio en los estilos de vida: siguieron las instrucciones y reglas más fácilmente, aunque el médico siempre buscaba controlarlo. Cuando hubo descontrol de la enfermedad, lo sujetos recibieron regaños y juzgamientos del médico y el resto de profesionales del equipo de salud, les decían: “¿Porqué no te cuidas?". Los sujetos sintieron que ir a la cita médica era "una mortificación. Da pena no hacer caso".

Como era de esperarse, la principal lucha en las personas con diabetes se evidenció frente a la prohibición de la comida. Una lucha entre el deber hacer racional y el querer hacer placentero:

Yo soy muy goloso y no he dejado de ser goloso con el pan; yo soy panadero a morir, y eso me hace mucho daño, como una harina es un azúcar, he tratado y he intentado dejarla, eso lo único que no me deja, digamos, estar tranquilo en el tema, pero no he podido, digamos que eso va a morir conmigo, lo estoy controlando [...] no lo quiero dejar. La verdad, no lo quiero dejar. (Roberto) 
Comer lo que les gusta es una manera de rebelarse y mantener el espacio propio, su capacidad de control, de decisión y su privacidad. La lucha se da más los fines de semana o en la noche, cuando reciben invitaciones o se sienten libres del deber, comen lo prohibido y lo preferido, y luego sienten culpa y hacen cambios. Se dan periodos alternados de cuidado/control y descuido/desorden (Cantarero, 2008).

Precisamente, para Michel De Certeau $(1999 ; 2000)$ las maneras de comer son complejas porque tienen una historia y surgen desde la infancia a partir de la relación con la madre, por lo tanto son difíciles de cambiar. Este planteamiento se confirmó en las dos mujeres entrevistadas, quienes al hablar de la comida, se remitieron al recuerdo de la primera infancia con su madre:

En mi familia, la comida ha sido una cosa especialísima. El recuerdo de mi mamá era haciendo oficio y haciendo de comer, nosotras hablamos con mi hermana de Estados Unidos y siempre nos acordamos de lo que mi mamá cocinaba; cocinaba, mejor dicho, delicioso; las mejores sopas del mundo las hacía ella. (Emma)

Nosotros con mi mamá, desde pequeñitos en la casa, nos acostumbraron a comer verduras, y nosotras no éramos tan gordas [...] mi mamá nos hacía frijoles, arroz, nos daba plato de ensalada y un maduro, pero cocinado, no frito, no sé si era por las circunstancias o la economía que era mi mamá. (Patricia)

Se muestra así una contraposición entre dos tipos de relación: entre la relación inicial con la madre, tendiente a regular nuestro cuerpo, ubicada entre el deber y el obedecer, y en una relación que proclama autonomía del sujeto sexuado, ubicada en la creación y la liberación. El reto será entonces, como lo plantea Michel De Certeau, promover la capacidad que tenemos los seres humanos de crear maneras propias de vivir, al margen de los mandatos, que nos permiten elegir e inventar formas de hacer cotidianas que adquieren un valor definitorio. 


\section{Conclusiones}

El cuerpo no puede concebirse en oposición de las dimensiones físico-biológicas y sociocultural, pues cuando el cuerpo enferma, se evidencia la compleja relación que existe entre la naturaleza y la cultura a partir del lugar simbólico que ocupa; su lenguaje y comunicación pone en evidencia la diversidad de aspectos que componen la condición humana: el dolor, el sufrimiento, el lugar del otro, la lucha por la autonomía frente a las determinaciones y la muerte. Ante la enfermedad, la experimentación corporal y la resultante noción del cuerpo cambia. No solo por las sensaciones corporales y subjetivas del ser humano, sino por las construcciones sociales que se hacen del cuerpo y de la enfermedad. En una enfermedad como la diabetes, la experiencia subjetiva puede vivenciarse como permanente en la cotidianidad de las personas, amenazando constantemente su vida, su salud y su autonomía por las regulaciones que tanto el mundo social como la mirada clínica buscan controlar.

La enfermedad también fue considerada como una invasión en el cuerpo que llegó de manera no esperada. Esta afecta el cuerpo y lo deteriora de manera progresiva sin poderlo evitar. La manera de concebir la enfermedad fue un reflejo de la condición histórico-social de las personas participantes y fue configurando las nociones que adquirió el cuerpo. Se reconoció la existencia de una dimensión fáctica del cuerpo, que se simboliza en las relaciones sociales y en la historia afectiva de las personas entrevistadas. En el caso de la enfermedad, esa dimensión fáctica del cuerpo simbolizada reflejó un componente de sufrimiento humano que se inserta en el proceso orgánico funcional.

Además, el discurso médico al que se vieron sometidas las personas con diabetes que participaron en la investigación se fue incorporando en la visión que construyeron sobre su cuerpo y la enfermedad; condicionándose así un tipo de cuidado que permitía, en ocasiones, cumplir con los preceptos clínicos y, en otras, ejecutar acciones "prohibidas" con la comida y el ejercicio que iban en contra de las posibles complicaciones que la enfermedad podría traerles. Este discurso promovió una visión organística y dividida en partes del cuerpo, cuya mirada biológica, funcionalista y medicalizada transcendía distintas esferas de la vida de las personas. Se enseña sobre la enfermedad para introducir al sujeto a las lógicas clínicas y someter al cuerpo a prácticas vigilantes y prohibitivas del deseo y el placer corporal. 


\section{Referencias}

Cantarero, A. L. (2008). Capítulo V. Género, estética corporal, alimentación y deporte. En Cuerpos que hablan. Montesinos Ensayo (pp. 143-170). España: Ediciones de Intervención Cultural.

Citro, S. (2009). Capitulo II. Variaciones sobre la corporalidad. En Cuerpos Significantes. Travesías de una etnografía dialéctica (pp. 43-82). Buenos Aires: Editorial Biblos.

De Certeau, M. (2000). La invención de lo cotidiano 1: Artes del Hacer. México: Universidad Iberoamericana. Instituto Tecnológico y de Estudios Superiores de Occidente.

De Certeau, M., Giard, L., y Mayol, P. (1999). La invención de lo cotidiano 2: Habitar y cocinar. México: Universidad Iberoamericana. Instituto Tecnológico y de Estudios Superiores de Occidente.

Elías, N. (1966). La sociedad cortesana. México: Fondo de Cultura Económica de México.

Escobar, M. C., y Sánchez, L. M. (2009). Mitos y Secretos Familiares. Cali: Colección Libros de Investigación. Programa Editorial Universidad del Valle.

Foucault, M. (1966). El nacimiento de la clínica. Una arqueología de la mirada médica. México: Siglo Veintiuno Editores S. A.

Foucault, M. (1990). Tecnologías del yo y otros textos afines. Barcelona: Ediciones Paidós Ibérica, S.A.

Foucault, M. (2006). Historia de la sexualidad. El cuidado de sí. (Vol 3). España: Siglo XXI.

Kleinman, A. (1988). The Illness Narratives. Suffering, Healing, and the Human Condition. United States of America: Basic Books.

Le Bretón, D. (1994). Lo imaginario del cuerpo en la tecnociencia. Revista Española de Investigaciones Sociológicas REIS (68), 197-210.

Le Bretón, D. (2007). Capítulo 1. Una antropología de los sentidos. En El sabor del mundo. Una antropología de los sentidos (pp. 19-49). Buenos Aires: Nueva Visión.

Le Bretón, D. (2007). Capitulo 2. De ver a saber. En El sabor del mundo. Una antropología de los sentidos (pp. 51 -91). Buenos Aires: Nueva Visión.

Mauss, M. (1979). Sociología y Antropología. Madrid: Editorial Tecnos.

Mendiola G. I. (Ed.). (2009). Regímenes de producción de cuerpos en el espacio médico. En Rastros y rostros de la biopolitica (pp. 223-246). Barcelona: Anthropos. 
Merleau-Ponty, M. (1984). Fenomenología de la percepción. Barcelona: Editorial Planeta - Agostini.

Ortega, A. F. (Ed). (1996). Lenguaje y cuerpo: transacciones en la construcción del dolor. En Sujetos del dolor, agentes de dignidad (pp. 343-373). Colombia: Colección Lecturas CES. Universidad Nacional de Colombia. Facultad de Ciencias Humanas. Centro de Estudios Sociales.

Pedraza, Z. (2008). Cuerpo e investigación en teoría social. Redalyc, 17(2), 247266. Recuperado de http://www.redalyc.org/pdf/122/12217203.pdf

Reyes, R. A. (2001). El devenir del discurso. Glosas para una interpretación del Cratil. Revista Fuentes Humanísticas (23), 93- 99. México: UAM Azcapotzalco.

Reyes, R. A., y Solana, E. (2008). El cuerpo y las ciencias sociales. Revista Pueblos y Fronteras digital (4), 1-56. Recuperado de http://www.pueblosyfronteras. unam.mx/a07n4/pdfs/n4_art02.pdf

Rolland, J. S. (2000). Familias, enfermedad y discapacidad. España: Gedisa Editorial.

Soler, C. (2003). Cuerpos Contemporáneos. El cuerpo, acontecimiento de discurso. Letrazas. Del Otro contemporáneo, sus crisis y sus urgencias (3), 62-68.

Suárez, R. (Comp.). (2001). Salud-enfermedad: una categoría a repensar desde la antropología. En Reflexiones en salud: una aproximación desde la antropología (11-21). Colombia: Ediciones Uniandes. Departamento de Antropología. Universidad de los Andes.

Tempone Pérez, S. G. 2007. El placebo en la práctica y en la investigación clínica. Anales de Medicina Interna, 24 (5), 249-252. Recuperado de http://dialnet. unirioja.es/servlet/articulo?codigo=2335005

Turner, B. S. (1989). El cuerpo y la sociedad. Exploraciones en teoría social. México: Fondo de Cultura Económica. 